\title{
On gauge invariance and the path integral
}

\author{
Silvio J. Rabello* and Carlos Farina \\ Instituto de Física \\ Universidade Federal do Rio de Janeiro \\ Rio de Janeiro RJ \\ Caixa Postal 68.528-CEP 21945-970 \\ Brasil
}

August 14, 2018

\begin{abstract}
Using a gauge covariant operator technique we deduce the path integral for a charged particle in an arbitrary stationary magnetic field, verifying the "midpoint rule" for the discrete form of the interaction term with the vector potential.
\end{abstract}

PACS numbers: 03.65.D, 11.15, 41.20 
The path integral for a charged particle in a magnetic field gives us a remarkable example of the subtleties of the discretized time approach and the relation between path integrals and Brownian motion. When one starts with the continuum definition of the path integral and tries to perform the functional integration by time slicing one is faced with the discretization of the term $\int d t \dot{\mathbf{x}} \cdot \mathbf{A}$, that is the interaction with the vector potential $\mathbf{A}(\mathbf{x})$, and that for each infinitesimal segment $\mathbf{x}_{n+1}-\mathbf{x}_{n}$ is given by $\left(\mathbf{x}_{n+1}-\mathbf{x}_{n}\right) \cdot \mathbf{A}(\mathbf{x})$. Naively one could think that it would not matter whether $\mathbf{A}(\mathbf{x})$ is evaluated at $\mathbf{x}_{n}, \mathbf{x}_{n+1}$ or somewhere in between. But it happens that for the path integral to satisfy the Schrödinger equation we must take the average of $\mathbf{A}(\mathbf{x})$ between $\mathbf{x}_{n}$ and $\mathbf{x}_{n+1}$ ("midpoint rule"). If we disregard this rule we are forced to introduce an extra term in the Schrödinger equation, breaking the gauge invariance. The reason for that is in the Brownian character of the paths in the functional integral [1]. Another approach to deduce the discretized path integral is to start from the Hamiltonian formulation of quantum mechanics and define it as an infinite product of operators where careful use of the Trotter formula for a proper " $\hat{\mathbf{x}}-\hat{\mathbf{p}}$ " ordering leads to the "midpoint rule" [2]. In this paper we present an alternative derivation of the gauge invariant path integral by applying a gauge covariant method developed long ago by Schwinger to study QED with background fields [3]. In this method instead of the " $\hat{\mathbf{x}}-\hat{\mathbf{p}}$ " ordering we use a time ordering procedure to obtain the configuration space path integral.

The Hamiltonian operator for a charged particle in an arbitrary stationary magnetic field is

$$
\hat{H}=\frac{\hat{\pi}^{2}}{2 m},
$$

with $\hat{\pi}^{i} \equiv \hat{p}^{i}-e \hat{A}^{i}(\mathrm{i}=1,2,3)$ obeying the algebra $(\hbar=1)$

$$
\left[\hat{\pi}_{i}, \hat{\pi}_{j}\right]=i e\left(\partial_{i} \hat{A}_{j}-\partial_{j} \hat{A}_{i}\right) \equiv i e \hat{F}_{i j}
$$

and the transition amplitude between the position eigenstates is given by

$$
\left\langle\mathbf{x}^{\prime \prime}, T \mid \mathbf{x}^{\prime}, 0\right\rangle=\left\langle\mathbf{x}^{\prime \prime}\left|e^{-i \hat{H} T}\right| \mathbf{x}^{\prime}\right\rangle
$$


One way to write a path integral for $\left\langle\mathbf{x}^{\prime \prime}, T \mid \mathbf{x}^{\prime}, 0\right\rangle$, is to decompose it according to Dirac [4] as

$$
\left\langle\mathbf{x}^{\prime \prime}, T \mid \mathbf{x}^{\prime}, 0\right\rangle=\int d \mathbf{x}_{N} \ldots \int d \mathbf{x}_{1}\left\langle\mathbf{x}^{\prime \prime}, T \mid \mathbf{x}_{N}, t_{N}\right\rangle\left\langle\mathbf{x}_{N}, t_{N} \mid \mathbf{x}_{N-1}, t_{N-1}\right\rangle \ldots\left\langle\mathbf{x}_{1}, t_{1} \mid \mathbf{x}^{\prime}, 0\right\rangle .
$$

Then we take $T=(N+1) \varepsilon$ and $t_{n}=n \varepsilon(n=1, \ldots, N)$ letting $N \rightarrow \infty$ and $\varepsilon \rightarrow 0$ with $\mathrm{T}$ fixed [5]. Next we obtain the small time propagator $\left\langle\mathbf{x}_{n+1}, t_{n}+\varepsilon \mid \mathbf{x}_{n}, t_{n}\right\rangle$, insert it in (4) and try to perform the infinite dimensional integral.

To evaluate the small time propagator we use a method developed by Schwinger in his early investigations on effective actions [3]. We now write the transition amplitude as $\left\langle\mathbf{x}^{\prime \prime}, T \mid \mathbf{x}^{\prime}, 0\right\rangle \equiv \exp (i W)$, where $W\left(\mathbf{x}^{\prime \prime}, \mathbf{x}^{\prime} ; T\right)$ is a complex function of the end point coordinates and time. Defining the expectation value of an observable $\hat{\mathcal{O}}$ by

$$
\langle\hat{\mathcal{O}}\rangle \equiv\left\langle\mathbf{x}^{\prime \prime}, T|\hat{\mathcal{O}}| \mathbf{x}^{\prime}, 0\right\rangle /\left\langle\mathbf{x}^{\prime \prime}, T \mid \mathbf{x}^{\prime}, 0\right\rangle
$$

it is easy to verify that $W$ is determined by the following equations

$$
\begin{aligned}
-\frac{\partial W\left(\mathbf{x}^{\prime \prime}, \mathbf{x}^{\prime} ; T\right)}{\partial T} & =\langle\hat{H}(\hat{\mathbf{x}}(T), \hat{\boldsymbol{\pi}}(T))\rangle \\
\frac{\partial W\left(\mathbf{x}^{\prime \prime}, \mathbf{x}^{\prime} ; T\right)}{\partial x_{i}^{\prime \prime}} & =\left\langle\hat{\pi}_{i}(T)\right\rangle+e A_{i}\left(\mathbf{x}^{\prime \prime}\right), \\
\frac{\partial W\left(\mathbf{x}^{\prime \prime}, \mathbf{x}^{\prime} ; T\right)}{\partial x_{i}^{\prime}} & =-\left\langle\hat{\pi}_{i}(0)\right\rangle-e A_{i}\left(\mathbf{x}^{\prime}\right), \\
W\left(\mathbf{x}^{\prime \prime}, \mathbf{x}^{\prime} ; 0\right) & =-i \ln \delta^{3}\left(\mathbf{x}^{\prime \prime}-\mathbf{x}^{\prime}\right) .
\end{aligned}
$$

To solve this problem Schwinger noticed that the above equations relate the transition amplitude to the solution of the Heisenberg equations for $\hat{\mathbf{x}}(T)$ and $\hat{\boldsymbol{\pi}}(T)$. If we solve for $\hat{\boldsymbol{\pi}}(T)$ in terms of $\hat{\mathbf{x}}(T)$ and $\hat{\mathbf{x}}(0)$ and insert this, in a time ordered fashion, on (6)-(8) we are left with a set of first order equations to integrate. For $\hat{\mathbf{x}}(\varepsilon)$ we have up to second order in $\varepsilon$

$$
\hat{x}^{i}(\varepsilon)=e^{i \hat{H} \varepsilon} \hat{x}^{i}(0) e^{-i \hat{H} \varepsilon} \simeq \hat{x}^{i}(0)+\frac{\hat{\pi}^{i}(0)}{m} \varepsilon+\frac{e}{4 m^{2}}\left(2 \hat{F}^{i k} \hat{\pi}_{k}(0)-i \partial_{k} \hat{F}^{i k}\right) \varepsilon^{2},
$$


with $\hat{F}^{i k}=\hat{F}^{i k}(\mathbf{x}(0))$. Inverting the above equation to get $\hat{\pi}^{i}(0)$ in terms of $\hat{\mathbf{x}}(\varepsilon)$ and $\hat{\mathbf{x}}(0)$

$$
\hat{\pi}^{i}(0) \simeq m \frac{\left(\hat{x}^{i}(\varepsilon)-\hat{x}^{i}(0)\right)}{\varepsilon}-\frac{e}{2}\left(\hat{x}^{k}(\varepsilon)-\hat{x}^{k}(0)\right) \hat{F}^{i k},
$$

using the fact that $\left\langle(\hat{\mathbf{x}}(\varepsilon)-\hat{\mathbf{x}}(0))^{2}\right\rangle$ is of order $\varepsilon$ [5], we see that if we take $\langle\hat{\boldsymbol{\pi}}(0)\rangle$ the terms in the above expansion are respectively of order $1 / \sqrt{\varepsilon}$ and $\sqrt{\varepsilon}$. The second term although small in comparison with the first, will give a relevant contribution when used in (7) and (8) to evaluate $W\left(\mathbf{x}_{n+1}, \mathbf{x}_{n} ; \varepsilon\right)$. From $\hat{\boldsymbol{\pi}}(0)$ we have by time evolution:

$$
\hat{\pi}^{i}(\varepsilon) \simeq m \frac{\left(\hat{x}^{i}(\varepsilon)-\hat{x}^{i}(0)\right)}{\varepsilon}+\frac{e}{2}\left(\hat{x}^{k}(\varepsilon)-\hat{x}^{k}(0)\right) \hat{F}^{i k} .
$$

Using the above expression for $\hat{\boldsymbol{\pi}}(0)$ or $\hat{\boldsymbol{\pi}}(\varepsilon)$ in $\hat{H}$, in a time ordered manner, we are ready to integrate (6)

$$
W\left(\mathbf{x}_{n+1}, \mathbf{x}_{n} ; \varepsilon\right) \simeq \frac{m\left(\mathbf{x}_{n+1}-\mathbf{x}_{n}\right)^{2}}{2 \varepsilon}+i \frac{3}{2} \ln \varepsilon+\Phi\left(\mathbf{x}_{n+1}, \mathbf{x}_{n}\right)
$$

where we used that $\left[\hat{x}^{i}(\varepsilon), \hat{x}_{i}(0)\right] \simeq-3 i \varepsilon / m$ and $\Phi$ is a time independent function of the end point coordinates $\mathbf{x}_{n+1}$ and $\mathbf{x}_{n}$. Inserting the above $W$ in (7) and (8) we have for $\Phi$ (remember $\left(\mathbf{x}_{n+1}-\mathbf{x}_{n}\right)^{2} \sim \varepsilon$ )

$$
\begin{aligned}
\frac{\partial \Phi}{\partial x_{n+1}^{i}} & =\frac{e}{2}\left[\left(x_{n+1}-x_{n}\right)^{k} \frac{\partial A_{k}\left(\mathbf{x}_{n+1}\right)}{\partial x_{n+1}^{i}}+A^{i}\left(\mathbf{x}_{n+1}\right)+A^{i}\left(\mathbf{x}_{n}\right)\right]+O(\varepsilon) \\
\frac{\partial \Phi}{\partial x_{n}^{i}} & =\frac{e}{2}\left[\left(x_{n+1}-x_{n}\right)^{k} \frac{\partial A_{k}\left(\mathbf{x}_{n}\right)}{\partial x_{n}^{i}}-\left(A^{i}\left(\mathbf{x}_{n+1}\right)+A^{i}\left(\mathbf{x}_{n}\right)\right)\right]+O(\varepsilon)
\end{aligned}
$$

with the solution

$$
\Phi\left(\mathbf{x}_{n+1}, \mathbf{x}_{n}\right)=\frac{e}{2}\left(\mathbf{x}_{n+1}-\mathbf{x}_{n}\right) \cdot\left(\mathbf{A}\left(\mathbf{x}_{n+1}\right)+\mathbf{A}\left(\mathbf{x}_{n}\right)\right)+C
$$

where $\mathrm{C}$ is a constant determined by (9)

$$
C=-i \frac{3}{2} \ln \left(\frac{m}{2 \pi i}\right) .
$$

Finally, the small time propagator is

$$
\left\langle\mathbf{x}_{n+1}, t_{n}+\varepsilon \mid \mathbf{x}_{n}, t_{n}\right\rangle \simeq\left(\frac{m}{2 \pi i \varepsilon}\right)^{\frac{3}{2}} e^{i S}
$$


with

$$
S=\frac{m\left(\mathbf{x}_{n+1}-\mathbf{x}_{n}\right)^{2}}{2 \varepsilon}+\frac{e}{2}\left(\mathbf{x}_{n+1}-\mathbf{x}_{n}\right) \cdot\left(\mathbf{A}\left(\mathbf{x}_{n+1}\right)+\mathbf{A}\left(\mathbf{x}_{n}\right)\right),
$$

that is precisely the midpoint expansion rule.

We see that by applying a gauge covariant method we were able to obtain the the "midpoint rule" in a natural way. In our deduction two facts were essential, first the Brownian like relation $\left(\mathbf{x}_{n+1}-\mathbf{x}_{n}\right)^{2} \sim \varepsilon$ and second the gauge covariance conditions (17) and (8). The authors are grateful to the CNPq (Brazilian Research Council) for the financial support.

\section{References}

[1] L.S. Schulman, Thecniques and Applications of Path Integration (John Wiley and Sons, New York, 1981).

[2] L.S. Schulman, in Lectures on Path Integration: Trieste 1991 (World Scientific, Singapore, 1993).

[3] J. Schwinger, Phys. Rev. 82, 664 (1951).

[4] P.A.M. Dirac, The Principles of Quantum Mechanics (3rd edn) (Oxford University Press, 1947).

[5] R.P. Feynman and A.R. Hibbs, Quantum Mechanics and Path Integrals (McGrawHill, New York, 1965). 